A N N A L E S Annales de Bretagne et des Pays de l'Ouest

\title{
Jonathan Dumont et Laure Fagnart (dir.), Georges Ier
} d'Amboise, 1460-1510

Une figure plurielle de la Renaissance

\section{Antoine Rivault}

\section{(2) OpenEdition}

\section{Journals}

Édition électronique

URL : http://journals.openedition.org/abpo/2889

DOI : $10.4000 /$ abpo.2889

ISBN : $978-2-7535-3977-8$

ISSN : 2108-6443

\section{Éditeur}

Presses universitaires de Rennes

\section{Édition imprimée}

Date de publication : 15 décembre 2014

Pagination : 182-184

ISBN : 978-2-7535-3975-4

ISSN : $0399-0826$

\section{Référence électronique}

Antoine Rivault, « Jonathan Dumont et Laure Fagnart (dir.), Georges ler d'Amboise, 1460-1510 », Annales de Bretagne et des Pays de

I'Ouest [En ligne], 121-4 | 2014, mis en ligne le 15 décembre 2014, consulté le 23 septembre 2020 URL : http://journals.openedition.org/abpo/2889 ; DOI : https://doi.org/10.4000/abpo.2889 
de France en 1422. Au cours de ces années, le destin de la capitale provinciale qu'est Poitiers s'entremêle, toutes proportions gardées, avec celui du royaume de France par la présence dans ses murs ou dans ses environs de ces deux personnages et de leur entourage. En toute logique l'index des noms de personnes livre de nombreuses occurrences à chacune des rubriques qui les concernent (p. 446 et 452). Il en est de même pour " Poitiers " dans l'index des noms de lieux, qui offre la rubrique de loin la plus fournie et la plus diverse (p. 432-436).

Grâce à un travail méticuleux de transcription, d'édition critique et d'indexation, Robert Favreau livre un abondant et riche matériel documentaire qui illustre les nombreuses études qu'il a lui-même réalisées sur la capitale du Poitou. Les historiens en tireront profit pour poursuivre les recherches locales comme pour développer des comparaisons avec d'autres villes. Enfin le lecteur curieux et érudit aura tout loisir de parcourir l'ouvrage en quête de menus faits, voire d'anecdotes qui éclairent certains aspects de la vie quotidienne d'une capitale provinciale vue au prisme des délibérations de ses magistrats municipaux (fonctionnement institutionnel et affaires de la commune, clergé et institutions ecclésiastiques, dont l'université, topographie de la ville et activités économiques...). La vision que l'on en retire est certes partielle et parfois réductrice, mais on ne peut que remercier Robert Favreau d'en avoir grandement facilité l'accès. On attend donc avec impatience la publication des autres registres en faisant nôtre la formule de Georges Duby : "L'histoire continue ".

Michel BOCHACA

Dumont, Jonathan, FAGNART, Laure (dir.), Georges Ier d'Amboise, 1460-1510. Une figure plurielle de la Renaissance, Rennes, PUR, coll. « Histoire », 2013, 274 p.

Ce livre est le résultat d'un colloque international organisé à l'université de Liège en 2010 à l'occasion du 500 anniversaire de la mort du cardinal Georges I Ier d'Amboise, archevêque de Rouen, légat a latere et principal conseiller du roi de France, Louis XII. La rencontre, placée sous le signe de l'interdisciplinarité, réunit 17 spécialistes du Moyen Âge tardif et du début du XvI ${ }^{\mathrm{e}}$ siècle. Les contributions forment 262 pages organisées en trois parties autour de la personne de Georges d'Amboise illustrée tour à tour par ses " actions et images politiques ", son rôle de " bâtisseur, bibliophile et amateur d'art " et enfin de sa " mise au tombeau à la memoria ". Dans une rapide introduction, Jonathan Dumont et Laure Fagnart font le point sur la très riche historiographie du cardinal, qui va du panégyrique à la diatribe.

La première partie, " Georges d'Amboise, actions et images politiques ", revient sur le rôle politique du cardinal du temps du règne de Louis XII. Ancien compagnon du temps où celui-ci n'était que duc d'Orléans, le cardinal d'Amboise devient son principal conseiller à son avènement à la couronne de France. Incarnation du prélat d'État (dans la France du début du Xvi ${ }^{\mathrm{e}}$ siècle, un conseiller du roi sur trois est un ecclésiastique), le cardinal d'Amboise, fortement associé à Louis XII, est dépeint comme "le premier des grands ministres-favoris de la France moderne " (Cédric Michon, p. 26). Laurent Vissière s'efforce de discerner autant que possible la philosophie politique du cardinal d'Amboise. Celle-ci reposait sur une stratégie du glacis et des États-tampons qui préservait la Pax Gallica. Porter la guerre en Milanais, malgré bien des échecs, c'était conserver la paix en France (p. 56). Ainsi Georges d'Amboise s'appuya-t-il sur des grands feudataires pour tenir la Lombardie française à l'image de Jean-Jacques Trivulce, gouverneur par intérim du Milanais (Marino Vigano). À l'intérieur du royaume de France, le cardinal avait une vision " moderne " et réfor- 
matrice. La rédaction des coutumes nécessitait ainsi une forte culture juridique que le cardinal s'efforça de faire reconnaître par les universités (Isabelle Gillet). Le livre fait également la part belle aux " images politiques " que véhiculait le cardinal comme des illustrations visuelles de sa puissance. L'héraldique et l'emblématique des membres de la famille d'Amboise sont ainsi finement analysées par Laurent Hablot qui note au passage que " par un heureux hasard " (p. 45), les couleurs de Georges d'Amboise correspondent à la fois aux couleurs personnelles de Louis XII (le jaune et le rouge) et à celles du gouvernement pontifical " dont elles adoptent l'exacte mise en forme palée ». Ainsi se dessine un cardinal « entre l'Église et l'État ", mais dont l'image de prélat d'État est davantage celle d'un prince temporel que celle du parfait ecclésiastique (Jonathan Dumont, p. 93). Par ailleurs, cette image fut à maintes reprises raillée par les contemporains du cardinal. Le théâtre de la Basoche, par exemple, le met en scène sous les traits du mauvais conseiller, manipulateur du roi (Nicole Hochner, p. 98). Sont également dénoncés les trop larges pouvoirs temporels du prélat et son goût pour le cumul des charges, des fonctions et des bénéfices.

La deuxième grande partie du livre s'attache à la figure d'important mécène que fut Georges d'Amboise. Archevêque de Rouen, le cardinal fit office de véritable bâtisseur en Normandie, supervisant les constructions du palais archiépiscopal de Rouen, le château de Déville et celui de Gaillon (Flaminia Bardati). C'est ce dernier qui concentre toute la magnificence du cardinal-ministre. Ce château longtemps décrit comme "premier foyer de la Renaissance en France ", célèbre pour son architecture et ses jardins, abritait également des décors intérieurs somptueux et de nombreuses œuvres d'art qui révèlent un fort attachement du prélat à Saint Jean-Baptiste (Laure Fagnart, p. 176). Des tableaux plus célèbres encore sont acheminés à Gaillon pour recevoir officiellement en 1508 le roi et la reine. En effet, à l'automne 1508, Georges d'Amboise y accueille Louis XII et les ambassadeurs étrangers (Xavier Pagazani). S'y déploie un discours politique par les arts dans lequel le cardinal n'hésite pas à représenter le roi en empereur romain (p. 168). Georges d'Amboise est également un important bibliophile. Toujours à Gaillon, sa bibliothèque ne rassemble pas moins de 250 ouvrages (mais assez peu d'imprimés), ce qui est considérable pour la France de l'époque (Marie-Pierre Laffitte). S'y côtoient des livres de droit, d'histoire, de liturgie et déjà des ouvrages de la Renaissance italienne. Son goût pour les arts, le cardinal aurait pu le transmettre à son neveu, Charles d'Amboise. Edoardo Villata convainc le lecteur que le gouverneur du duché de Milan, au lieu d'être le rude militaire que nous connaissons, pourrait avoir été " un connaisseur très perspicace " de l'art italien de l'époque, capable de reconnaître de futurs talents comme Le Corrège (p. 207). Par ailleurs, à Gaglianico (Biella), les fresques de la chapelle du château rendent hommage à Charles d'Amboise (Vittorio Natale). Ainsi, c'est bien " le clan Amboise " qui affirme son goût pour les arts.

Enfin, une brève troisième partie (deux contributions) se penche sur la memoria du cardinal après sa mort survenue en mai 1510 . En faisant le pari de faire une comparaison de ses funérailles rouennaises avec celle d'un autre proche de Louis XII, Gaston de Foix, Alain Marchandisse montre bien l'originalité des cérémonies funéraires du cardinal. Bien qu'issu d'une famille moins illustre que celle de Gaston de Foix, Georges d'Amboise bénéficie d'obsèques quasi royales du fait de la présence d'une effigie (p. 236). Enfin, la mise en bière du cardinal dans son église métropolitaine de Rouen lui offre une "vie posthume " (Gabriela Reuss). Son imposant monument funéraire dédié à la Vierge (qu'il partage avec un autre neveu) présente une effigie " animée " du cardinal " qui se trouve ainsi à la jonction entre la présence réelle du corps et sa représentation " (p. 246).

Pour reprendre à quelques mots près une formule de la conclusion (Franz Bierlaire), ce colloque consacré à Georges ${ }^{\text {er }}$ d'Amboise s'efforce d'abattre 
le mur entre "Gothique " et " Renaissance ". À travers la vie du cardinal d'Amboise se dessine bien une " figure plurielle de la Renaissance ". Si certains axes ont été privilégiés, il n'en reste pas moins que l'ouvrage présente des articles variés, qui se complètent davantage qu'ils ne s'additionnent. Preuve s'il en est besoin de la pertinence de l'interdisciplinarité y compris dans une approche biographique.

Antoine RIVAULT

HAUDRÈre, Philippe, Les Français dans l'océan Indien, XVII'-XIXe siècle, coll. "Histoire ", Rennes, PUR, 2014, 330 p.

Est-il besoin de présenter ici l'auteur du présent ouvrage, Philippe Haudrère, professeur émérite à l'université d'Angers et éminent spécialiste des compagnies des Indes depuis la soutenance puis la publication de sa thèse d'état sur La compagnie française des Indes au XVIII e siècle (1719-1795), en 1989? Depuis, Philippe Haudrère n'a cessé de produire des articles et ouvrages sur des sujets connexes, et l'on citera plus particulièrement sa synthèse sur Les compagnies des Indes Orientales (Paris, Desjonquères, 2006). Bien malheureusement, ses multiples articles originaux restent souvent épars, insérés dans des publications parfois difficiles à retrouver et consulter. Le présent ouvrage remédie à cela en proposant l'édition ordonnée d'une sélection de vingt-neuf articles, qui offre une première synthèse des écrits de Philippe Haudrère, redonnant ainsi toute sa cohérence à une œuvre historique magistrale, qui s'est déployée sur plus de quarante ans. La bibliographie proposée en annexe permet en outre au lecteur de disposer d'un panorama complet des travaux de l'auteur (p. 315-321). Les articles présentés comportent cartes (copies de documents originaux ou outils d'analyse), illustrations, graphiques et tableaux statistiques, dont on trouvera la table p. 323-324.

Ces articles sont ordonnés selon quatre thèmes. La première partie - Les compagnies des Indes ( 8 articles) - se concentre sur l'organisation des compagnies, en mettant l'accent sur les principaux acteurs (chapitres II, III, IV) avant de se concentrer sur les produits (chapitres VI, VII) tout en mettant en lumière le caractère structurel d'économie mixte de ce secteur d'activité.

Après avoir posé les cadres du développement de cette aventure, on peut emprunter, avec Philippe Haudrère, la Route des Indes (deuxième partie, 7 articles). Ce qui frappe immédiatement est la dangerosité : piraterie (chapitre IX), guerres (chapitre XI), scorbut et autres maladies dues aux conditions d'hygiène déplorables (chapitre XII), naufrages (chapitre XIII) constituent le quotidien de ceux qui tentèrent l'aventure de l'océan Indien. Pour contrer ces aléas, la compagnie s'organise : elle fait construire ses propres vaisseaux à Lorient à partir de 1729 (chapitre x) et se dote ainsi d'une solide flotte de commerce avant d'envisager le développement d'une flotte de guerre (chapitre XI). Elle améliore aussi sa connaissance de la région en confiant à d'Après de Mannevillette la réalisation d'une carte marine (chapitre XIV) corrigeant les erreurs des exemplaires français et hollandais en usage. Elle fait enfin de ses officiers de vaisseaux un corps d'élite de la Marine française (chapitre Xv).

Tout ceci rend possible la présence et l'activité européenne aux Indes Orientales, dont la troisième partie étudie divers aspects. Après avoir découvert les sources de la connaissance des Indes Orientales en Europe (récits de commerçants, lettres de missionnaires et cartes des marins et géographes sont présentés au chapitre $\mathrm{xVI}$ ), le lecteur entrevoit la vie outre-mer à travers la présentation de la monnaie à Pondichéry (chapitre XvIII) ou l'étude de ceux qui réussirent, qu'il s'agisse de la 